\title{
Efeitos de maturadores nas características tecnológicas da cana-de-açúcar com e sem estresse hídrico
}

\section{The effect of maturators on technological characterists of sugar cane on soils with and without water stress}

\author{
José Carlos Vieira de Almeida ${ }^{1}$; Célio Roberto F. Leite ${ }^{2}$; José Roberto Pinto de Souza ${ }^{1}$
}

\section{Resumo}

A cana-de-açúcar é uma das principais plantas cultivadas no Brasil. A maioria das áreas plantadas com cana-de-açúcar na região Centro-Sul do Brasil está sujeita à ocorrência do florescimento, o que não é desejado uma vez que este reduz o teor de sacarose. O objetivo do experimento foi avaliar o uso de maturadores nas características tecnológicas da cana soca em solo com e sem estresse hídrico. O delineamento experimental utilizado foi fatorial $2 \times 4$ (duas condições de umidade do solo e três tratamentos com maturadores mais uma testemunha) em blocos casualizados com 4 repetições. Os maturadores utilizados foram sulfometuron-methyl $\left(15 \mathrm{~g} \mathrm{ha}^{-1}\right)$ aplicado com e sem surfactante e o ethephon (480g ha ${ }^{1}$ ). As áreas úteis foram compostas por 3 linhas de cana da variedade reagente SP $813250 \mathrm{com} 8 \mathrm{~m}$ de comprimento espaçadas de 1,40 m entre elas. As características avaliadas foram: Pol\%, fibra e Brix aos 0 , 21, 30, 45, 60 e 75 dias após aplicações (DAA). A ação dos maturadores aumentou significativamente com a umidade do solo a 0 e 21 dias após a aplicação. Os aumentos de Pol\% teor de fibra e Brix não foram significativos com a aplicação de sulfometuron-methyl e ethephon. A aplicação de sulfometuron-methyl antecipou a colheita da cana soca em 15 dias.

Palavras-chave: Florescimento, reguladores de crescimento, Saccharum officinarum

\begin{abstract}
The sugar cane is one of the most important crop in Brazil. Many areas cultivated with this crop is subjected to flowering, which is not wanted once it reduces sucrose. The objetive of the present trial was to evaluate the effect of maturators on technological characterists of sugar cane on soils with and without water stress. The experimental design was a $2 \times 4$ factorial (two water conditions in the soil, three maturators and control) replicated four times. The maturators were sulfometuron-methil (15 $\left.\mathrm{g} \mathrm{ha}^{-1}\right)$ aplied with and whithout adjuvants and ethephon $\left(480 \mathrm{~g} \mathrm{ha}^{-1}\right)$. The evaluated area was arranged by three lines of sugar cane of the reagent cultivar SP 813250, with 8 meter long spaced in 1,4 m between rows. The evaluated characteristics were Pol\%, fibre and Brix at 0,21,30, 45, 60 and 75 days after aplication. The maturator action increased significant with the soil water at 0 and 21 days after aplication. The increases in Pol\%, fiber and Brix were not significant with the application of sulfometuron-methil and ethephon. The application of sulfometuron-methil antecipated the harvest of sugar in 15 days.
\end{abstract}

Key words: Flowering, growth regulator, Saccharum officinarum

1 Professor Adjunto, Depto de Agronomia, C.C.A., Universidade Estadual de Londrina (UEL), C.P. 6001, CEP 86.051-990. Londrina - PR. E-mail: vieira@laborsolo.com.br

2 Engenheiro Agrônomo, M.Sc., doutorando do Curso de Pós Graduação em Agronomia, Depto de Agronomia, C.C.A., UEL, C.P. 6001, CEP 86.051-990, Londrina - PR

* Autor para correspondência.

Recebido para publicação 02/05/05 Aprovado em 26/08/05 


\section{Introdução}

A cana-de-açúcar é uma das principais plantas cultivadas e encontra-se entre as de cultivo mais antigo no Brasil. Historicamente, as pesquisas agrícolas têm sido voltadas principalmente para a obtenção de maiores incrementos na produção total da cultura e extração de seus produtos e subprodutos a partir do colmo (açúcar, álcool, celulose e biogás) (MARTINS; CASTRO, 1999).

Dentro do complexo sistema de produção da indústria açucareira, a maturação da cana-de-açúcar é um dos aspectos mais importantes, pois é dele que depende o fornecimento de um fluxo contínuo de matéria prima para o funcionamento constante da usina durante o período de colheita. Sob uma perspectiva econômica e dentro da prática agronômica, a cana é considerada madura, ou em condição de ser industrializada, quando apresentar teor mínimo de sacarose (Pol\% da cana) acima de $12,275 \%$ do peso do colmo, sendo melhor o rendimento quanto maior for esta variável (DEUBER, 1988). Em algumas regiões, como por exemplo no Estado do Paraná, muitas vezes as condições de precipitação e temperatura nos meses de abril, maio e junho, que correspondem ao início da safra, favorecem o desenvolvimento vegetativo da cana, em detrimento do acúmulo de sacarose, retardando a possibilidade de atingir os valores mínimos; conseqüentemente, tem-se matéria prima de baixa qualidade tecnológica ou mesmo a falta desta para o adequado funcionamento da indústria (RODRIGUES, 1995).

Há incentivo a utilização de técnicas para aumentar a qualidade tecnologica da matéria prima destinada à indústria, como a aplicação de reguladores de vegetais. Com o uso do maturador é possível se obter dois resultados positivos: antecipação de corte e aumento do percentual de sacarose, quando se considera a cana em fase de desenvolvimento vegetativo. Os reguladores vegetais agem alterando a morfologia e a fisiologia da planta, podendo levar à modificações qualitativas e quantitativas na produção (MARTINS; CASTRO, 1999). Diversos produtos químicos, como glyphosate, etil-trimexapac, paraquat, fluazifop-p-butil e ethephon, além do sulfometuron-methyl, estão registrados no Brasil para o uso como maturador ou regulador crescimento vegetal. Destes, o mais tradicional é o ethephon, que reduz o florescimento, eleva os teores de sacarose e não propicia a morte da região apical; promove apenas uma redução de crescimento no entrenó em formação no momento da aplicação (CASTRO et al., 2001). Outros como o glyphosate (180 $\left.\mathrm{g} \mathrm{ha}^{-1}\right)$ e fluazifop-p-buthyl $\left(65 \mathrm{~g} \mathrm{ha}^{-1}\right)$ promovem a morte da região apical, tornando obrigatório que a colheita seja realizada dentro de um intervalo máximo após a aplicação, pois ocorre inversão da sacarose nas regiões vizinhas às necrosadas. O sulfometuronmethyl (15 $\left.\mathrm{g} \mathrm{ha}^{-1}\right)$ já foi usado como maturador em cana-de-açúcar (OLIVEIRA; CASTRO et al., 1993; CONSTANTIN et al., 2000), antecipando em 15 dias a colheita. Este produto, assim como o ethephon, não promove a morte da região apical nas doses utilizadas como maturador.

Gerenciamento da colheita da cana com segurança, e o melhor benefício por hectare é o que todo produtor espera de um maturador. Ele aumenta o teor de sacarose (POL\% da cana), melhora o período útil de industrialização(PUI), inibe o florescimento e reduz a isoporização, sem afetar a produtividade e a brotação da soqueira (CASAGRANDE, 1991).

A maioria das áreas plantadas com cana-deaçúcar na região Centro-Sul do Brasil está sujeita à ocorrência do florescimento. A intensidade do processo de florescimento e as conseqüências na qualidade da matéria-prima variam com a variedade e com o clima (SALATA; FERREIRA, 1977). Portanto, o uso de maturadores tem provocado resultados erráticos como brotações e diminuição dos internódios, em função de fatores do meio como temperatura e índice de precipitação.

Em função do exposto, o objetivo do presente experimento foi avaliar o uso de maturadores nas 
características tecnológicas da cana soca na região sucroalcooleira do Norte do Paraná em solo com e sem estresse hídrico.

\section{Material e Métodos}

O presente experimento foi conduzido no ano agrícola de 2001, na Fazenda Nova Maragogipe, no município de Jaquapitã, PR, situada na latitude de $23^{\circ} 05^{\prime} 33^{\prime \prime} \mathrm{S}$ e longitude de $51^{\circ} 35^{\prime} 02^{\prime}$ 'W, em condições de clima classificado como Cfa na escala de Köeppen. A temperatura média do mês mais quente é de 27 a $28^{\circ} \mathrm{C}$ e a temperatura média do mês mais frio, de 16 a $17^{\circ} \mathrm{C}$. A precipitação média do mês menos chuvoso é de 200 a $225 \mathrm{~mm}$ e a precipitação média do mês mais chuvoso, de 500 a $600 \mathrm{~mm}$ (CORREA; GODOY; BERNARDES, 1982). O solo da área experimental foi classificado como Latossolo Vermelho distroférrico (EMPRESA BRASILEIRA DE PESQUISA AGROPECUARIA, 1999).

O delineamento experimental utilizado foi um fatorial $2 \times 4$ em blocos casualizados com 4 repetições. $\mathrm{O}$ fator 1 refere-se às duas épocas de aplicação, sendo a primeira época em condições climáticas pouco favoráveis e estresse hídrico, e segunda em melhores condições climáticas, isto é, sem estresse hídrico. $\mathrm{O}$ fator 2 refere-se aos três tratamentos com maturadores mais uma testemunha para cada época de aplicação. Os maturadores utilizados foram sulfometuron-methyl aplicado com e sem surfactante e o ethephon. Todos os tratamentos aplicados estão descritos na Tabela 1. As parcelas experimentais foram compostas por 5 linhas de cana de $12 \mathrm{~m}$ de comprimento cada uma espaçadas de 1,40 m entre elas, e a área útil foi composta por três linhas centrais de $8 \mathrm{~m}$ de comprimento cada uma.

Tabela 1. Tratamentos utilizados para controle do florescimento da cana soca de segundo corte. Jaguapitã, PR. 2001.

\begin{tabular}{|c|c|c|c|c|}
\hline Tratamentos & $\begin{array}{l}\text { Maturador } \\
\left(\mathrm{g} \mathrm{ha}^{-1}\right)\end{array}$ & $\begin{array}{l}\text { Óleo Mineral } \\
\qquad\left(v^{-1}\right)\end{array}$ & $\begin{array}{l}\text { Surfactante } \\
\text { Não-Iônico } \\
\left(\mathbf{v ~ v}^{-1}\right)\end{array}$ & Solo \\
\hline $\mathrm{T} 1$ & Não Aplicado & Não Aplicado & Não Aplicado & $\operatorname{Seco}\left(E_{1}\right)$ \\
\hline $\mathrm{T} 2$ & Não Aplicado & Não Aplicado & Não Aplicado & Umido $\left(\mathrm{E}_{2}\right)$ \\
\hline $\mathrm{T} 3$ & $\begin{array}{l}\text { Sulfometuron-methyl } \\
\quad(15 \text { UNIDADE })\end{array}$ & Assist $0,1 \%$ & Não Aplicado & $\operatorname{Seco}\left(E_{1}\right)$ \\
\hline $\mathrm{T} 4$ & $\begin{array}{l}\text { Sulfometuron-methyl } \\
\quad(15 \text { UNIDADE })\end{array}$ & Assist $0,1 \%$ & Não Aplicado & Umido $\left(\mathrm{E}_{2}\right)$ \\
\hline T5 & $\begin{array}{l}\text { Sulfometuron-methyl } \\
\text { (15 UNIDADE) }\end{array}$ & Não Aplicado & Extravon $0,1 \%$ & $\operatorname{Seco}\left(E_{1}\right)$ \\
\hline T6 & $\begin{array}{l}\text { Sulfometuron-methyl } \\
\text { (15 UNIDADE) }\end{array}$ & Não Aplicado & Extravon $0,1 \%$ & Umido $\left(\mathrm{E}_{2}\right)$ \\
\hline $\mathrm{T} 7$ & $\begin{array}{c}\text { Ethephon } \\
\text { [ácido (2-cloroetil) fosfônico] } \\
\text { (480 UNIDADE) }\end{array}$ & Não Aplicado & Não Aplicado & $\operatorname{Seco}\left(E_{1}\right)$ \\
\hline $\mathrm{T} 8$ & $\begin{array}{c}\text { Ethephon } \\
\text { [ácido (2-cloroetil) fosfônico] } \\
\text { (480 UNIDADE) }\end{array}$ & Não Aplicado & Não Aplicado & Umido $\left(\mathrm{E}_{2}\right)$ \\
\hline
\end{tabular}

Semina: Ciências Agrárias, Londrina, v. 26, n. 4, p. 441 -448, out./dez. 2005 
Os tratamentos foram aplicados sobre cana soca de segundo corte da variedade reagente SP813250 desenvolvida pela Copersucar.

Os tratamentos T3, T5 e T7 foram aplicados em 16/03/2001 $\left(E_{1}\right)$. Neste dia, as condições climáticas se apresentavam com ausência de nebulosidade, umidade relativa do ar de 50,0\%, temperatura de $33,7^{\circ} \mathrm{C}$ e solo seco, $-30 \mathrm{KPa}$ detectado pelo tensiometro localizado na zona efetiva do sistema radicular.

Os tratamentos T4, T6 e T8 foram aplicados em $22 / 03 / 2001\left(E_{2}\right)$, uma semana após a primeira época. Neste período houve precipitações que totalizaram $38 \mathrm{~mm}$ até a aplicação dos tratamentos, o solo estava úmido (10KPa detectado pelo tensiometro localizado na zona efetiva do sistema radicular), a umidade relativa do ar de $61,0 \%$, a temperatura de $29,5^{\circ} \mathrm{C}$ e o céu com ausência de nebulosidade.

Para aplicação dos tratamentos foi utilizado um pulverizador costal propelido a $\mathrm{CO}_{2}$, com pressão constante de $310 \mathrm{KPa}$, equipado com bicos Teejet 110:01, o que proporcionou um volume de $100 \mathrm{~L}$ de calda ha-1 . A barra de aplicação foi mantida a $0,50 \mathrm{~m}$ acima das plantas de cana-de-açúcar, através do uso de montante laterais de 2,5 $\mathrm{m}$ de altura, uma vez que as plantas se encontravam em fase de pré-maturação.

Em todas as coletas foram encaminhados 15 colmos por parcela para avaliação de porcentagem de Pol, fibras e Brix, as quais foram realizadas aos 0 , 21, 30, 45, 60 e 75 dias após aplicações (DAA). O Pol, teor de fibra e Brix foram determinado segundo as normas operacionais definidas pela CONSECANA-SP (ORPLANA, 2005). Após as avaliações, foram calculadas as diferenças entre os tratamentos e a testemunha quando considerandose como ponto de partida o dia da aplicação.

Todos os dados colhidos foram submetidos à análise de variância e a comparação entre as médias foi realizada pelo teste de Tukey no nível de 5\% de probabilidade.

\section{Resultados e Discussão}

A colheita realizada ao 0 DAA na época $E_{2}$ provocou acréscimo significativo de Pol\% e Brix da cana como pode ser observado nas Tabelas 2 e 3.

Tabela 2. Dados de Pol\% de caldo de cana soca obtidos com a aplicação de dois maturadores em dois níveis de umidade do solo em seis épocas de avaliação. Jaguapitã, PR. 2001.

\begin{tabular}{|c|c|c|c|c|c|c|c|c|c|c|c|c|}
\hline \multirow[t]{2}{*}{ Tratamento } & \multicolumn{2}{|c|}{0 DAA } & \multicolumn{2}{|c|}{21 DAA } & \multicolumn{2}{|c|}{30 DAA } & \multicolumn{2}{|c|}{45 DAA } & \multicolumn{2}{|c|}{60 DAA } & \multicolumn{2}{|c|}{75 DAA } \\
\hline & Seco & Umido & Seco & Umido & Seco & Umido & Seco & Umido & Seco & Umido & Seco & Umido \\
\hline $\mathrm{T}$ & $\begin{array}{c}9,9 \mathrm{a} \\
\mathrm{B}\end{array}$ & $\begin{array}{c}10,6 \\
\text { A }\end{array}$ & $\begin{array}{c}11,2 b \\
B\end{array}$ & $\begin{array}{c}12,0 \\
\text { A }\end{array}$ & $\begin{array}{c}12,5 \mathrm{~b} \\
\mathrm{~A}\end{array}$ & $\begin{array}{c}13,2 \mathrm{a} \\
\mathrm{A}\end{array}$ & $\begin{array}{c}12,8 \mathrm{a} \\
\mathrm{A}\end{array}$ & $\begin{array}{r}13,1 \\
\mathrm{~A}\end{array}$ & $\begin{array}{c}13,3 \mathrm{a} \\
\mathrm{A}\end{array}$ & $13,7 \mathrm{a}$ & $\begin{array}{c}13,5 \mathrm{a} \\
\mathrm{A}\end{array}$ & $13,2 \mathrm{a}$ \\
\hline & $10,0 \mathrm{a}$ & $11,0 \mathrm{a}$ & $11,8 \mathrm{ab}$ & $12,4 \mathrm{a}$ & $13,3 \mathrm{ab}$ & $13,6 \mathrm{a}$ & $13,2 \mathrm{a}$ & $13,9 \mathrm{ab}$ & $13,6 \mathrm{a}$ & $13,6 \mathrm{a}$ & $13,9 \mathrm{a}$ & $14,0 \mathrm{a}$ \\
\hline & $\mathrm{B}$ & A & $A$ & & $\Delta$ & & 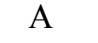 & A & A & A & A & \\
\hline$T$ & $\begin{array}{c}0,3 \mathrm{a} \\
\mathrm{A}\end{array}$ & $\begin{array}{c}11,0 \mathrm{a} \\
\mathrm{A}\end{array}$ & $\begin{array}{c}12,3 \mathrm{a} \\
\mathrm{A}\end{array}$ & $\begin{array}{c}12,4 \mathrm{a} \\
\mathrm{A}\end{array}$ & $\begin{array}{c}13,6 \mathrm{a} \\
\mathrm{A}\end{array}$ & $\begin{array}{c}13,5 \mathrm{a} \\
\mathrm{A}\end{array}$ & $\begin{array}{c}13,3 \mathrm{a} \\
\mathrm{A}\end{array}$ & $\begin{array}{c}13,9 \mathrm{a} \\
\mathrm{A}\end{array}$ & $\begin{array}{c}13,7 \mathrm{a} \\
\mathrm{A}\end{array}$ & $\begin{array}{c}13,4 \mathrm{a} \\
\mathrm{A}\end{array}$ & $\begin{array}{c}14,2 \text { a } \\
\text { A }\end{array}$ & $\begin{array}{c}14,3 \text { a } \\
\text { A }\end{array}$ \\
\hline & $\begin{array}{c}9,7 \mathrm{a} \\
\mathrm{B}\end{array}$ & $\begin{array}{c}10,7 \mathrm{a} \\
\mathrm{A}\end{array}$ & $\begin{array}{c}11,2 \mathrm{~b} \\
\mathrm{~B}\end{array}$ & $\begin{array}{c}12,0 \mathrm{a} \\
\mathrm{A}\end{array}$ & $\begin{array}{c}12,4 \quad b \\
A\end{array}$ & $\begin{array}{c}12,9 \mathrm{a} \\
\mathrm{A}\end{array}$ & $\begin{array}{c}12,7 \text { a } \\
\text { A }\end{array}$ & $\begin{array}{c}12,8 \mathrm{~b} \\
\mathrm{~A}\end{array}$ & $\begin{array}{c}13,8 \mathrm{a} \\
\mathrm{A}\end{array}$ & $\begin{array}{c}13,2 \mathrm{a} \\
\mathrm{A}\end{array}$ & $\begin{array}{c}13,2 \\
\mathrm{~A}\end{array}$ & $\begin{array}{c}4,1 \mathrm{a} \\
\mathrm{A}\end{array}$ \\
\hline
\end{tabular}

Médias seguidas das mesmas letras minúsculas na coluna e maiúsculas na linha não diferem significativamente no nível de 5\% de probabilidade pelo Teste de Tukey. 
Tabela 3. Dados de teor de fibra (\%) da cana soca obtidos com a aplicação de dois maturadores em dois níveis de umidade do solo em seis épocas de avaliação. Jaguapitã, PR. 2001

\begin{tabular}{|c|c|c|c|c|c|c|c|c|c|c|c|c|}
\hline \multirow[t]{2}{*}{ Tratamento } & \multicolumn{2}{|c|}{0 DAA } & \multicolumn{2}{|c|}{21 DAA } & \multicolumn{2}{|c|}{ 30 DAA } & \multicolumn{2}{|c|}{45 DAA } & \multicolumn{2}{|c|}{ 60 DAA } & \multicolumn{2}{|c|}{75 DAA } \\
\hline & Seco & Umido & Seco & Umido & Seco & Umido & Seco & Umido & Seco & Umido & Seco & Umido \\
\hline T1 e T2 & $8,9 \mathrm{a}$ & $9,3 \mathrm{a}$ & $9,3 \mathrm{a}$ & $9,7 \mathrm{a}$ & $9,9 \mathrm{a}$ & $10,0 \mathrm{a}$ & $10,6 \mathrm{a}$ & $9,7 \mathrm{a}$ & $10,2 \mathrm{a}$ & $9,7 \mathrm{a}$ & $11,6 \mathrm{a}$ & $11,4 \mathrm{a}$ \\
\hline & A & A & A & A & A & A & A & B & A & A & A & A \\
\hline $\mathrm{T} 3$ e $\mathrm{T} 4$ & $9,2 \mathrm{a}$ & $9,5 \mathrm{a}$ & $9,6 \mathrm{a}$ & $9,7 \mathrm{a}$ & $10,2 \mathrm{a}$ & $10,5 \mathrm{a}$ & $10,7 \mathrm{a}$ & $10,5 \mathrm{a}$ & $10,2 \mathrm{a}$ & $10,2 \mathrm{a}$ & $11,1 \mathrm{a}$ & $11,9 \mathrm{a}$ \\
\hline & A & A & A & A & A & A & A & A & A & A & A & $A$ \\
\hline T5 e T6 & $8,8 \mathrm{a}$ & $9,9 \mathrm{a}$ & $9,8 \mathrm{a}$ & $9,4 \mathrm{a}$ & $9,9 \mathrm{a}$ & $10,0 \mathrm{a}$ & $10,4 \mathrm{a}$ & $10,1 \mathrm{a}$ & $10,8 \mathrm{a}$ & $9,7 \mathrm{a}$ & $10,7 \mathrm{a}$ & $11,3 \mathrm{a}$ \\
\hline & A & A & A & A & A & A & A & A & A & A & A & \\
\hline & $\begin{array}{c}9,0 \mathrm{a} \\
\mathrm{A}\end{array}$ & $\begin{array}{c}9,7 \mathrm{a} \\
\mathrm{A}\end{array}$ & $\begin{array}{c}9,4 \mathrm{a} \\
\mathrm{A}\end{array}$ & $\begin{array}{c}9,4 \mathrm{a} \\
\mathrm{A}\end{array}$ & $\begin{array}{c}10,0 \mathrm{a} \\
\mathrm{A}\end{array}$ & $\begin{array}{c}10,4 \mathrm{a} \\
\mathrm{A}\end{array}$ & $\begin{array}{c}10,4 \mathrm{a} \\
\mathrm{A}\end{array}$ & $\begin{array}{c}9,8 \mathrm{a} \\
\mathrm{A}\end{array}$ & $\begin{array}{c}10,7 \mathrm{a} \\
\mathrm{A}\end{array}$ & $\begin{array}{c}10,0 \mathrm{a} \\
\mathrm{A}\end{array}$ & $\begin{array}{c}11,3 \mathrm{a} \\
\mathrm{A}\end{array}$ & $\begin{array}{c}11,2 \mathrm{a} \\
\mathrm{A}\end{array}$ \\
\hline
\end{tabular}

Médias seguidas das mesmas letras minúsculas na coluna e maiúsculas na linha não diferem significativamente no nível de 5\% de probabilidade pelo Teste de Tukey.

As condições climáticas do dia 16/03/2001 $\left(\mathrm{E}_{1}\right)$ não se apresentavam favoráveis para a aplicação e a ação dos produtos. Já para o dia 22/03/2001 $\left(\mathrm{E}_{2}\right)$, as condições climáticas apresentavam-se mais favoráveis para a aplicação e ação dos produtos (Tabelas 2, 3 e 4 ).

Houve aumento gradual do Pol\% no decorrer do tempo em todos os tratamentos em função da maturação natural da cana, o que deve estar relacionado com estresse hídrico, baixa precipitação e diminuição da temperatura no período. Todos os tratamentos tiveram comportamento semelhante no período das avaliações. Os maiores incrementos em Pol\% foram verificados nos tratamentos com sulfumeturon-methyl (Tabela 2). Este comportamento demonstra o efeito aditivo do produto em apressar a maturação e consequentemete antecipar a colheita.

Aos 21 DAA, os tratamentos com sulfometuronmethyl foram os que promoveram os maiores acréscimos de Pol\%, tanto na primeira época $\left(E_{1}\right)$ como na segunda $\left(E_{2}\right)$, com valores maiores que a testemunha e o ethephon, embora sem ainda ter atingido o valor mínimo de $13,0 \%$ exigido pela indústria. Aos $30 \mathrm{DAA}$, somente os tratamentos com sulfometuron-methyl, tanto em $\mathrm{E}_{1}$ como $\mathrm{E}_{2}$, atingiram o índice mínimo exigido pela indústria que é de 13,0\% de Pol\%. Ressalta-se ainda que o desempenho com a aplicação do surfactante foi superior ao óleo mineral. Aos 45 DAA, os índices de açúcar nos tratamentos com ethephon ainda eram inferiores a $13,0 \%$ para as duas épocas.

A aplicação de sulfometuron-methyl provocou aumento mínimo de 0,46 no Pol\%, enquanto o experimento de Oliveira et al. (1993) verificaram que a aplicação de sulfometuron metil $\left(15 \mathrm{~g} \mathrm{ha}^{-1}\right) \mathrm{e}$ ethephon (480 $\mathrm{g} \mathrm{ha}^{-1}$ ) proporciona aumento de no mínimo 1,12 no Pol \% cana. Nas condições do experimento, detectou-se uma antecipação de 15 dias na colheita da cana-soca, o indice mínimo exigido pela indústria foi atingido, quando aplicado o sulfometuron-methyl na primeira época. Resultados semelhantes foram obtidos por Oliveira e Castro (1993), Constantin, Maciel e Contiero (2000) que verificaram antecipação de 15 dias na colheita com a aplicação de sulfometuron-methyl (15 $\left.\mathrm{g} \mathrm{ha}^{-1}\right)$. Já aplicação de Ethrel 240 (ácido 2-cloroetilfosfônico) $2 \mathrm{~L}$ ha-1 e Arvest 480 (ethephon) $1 \mathrm{~L} \mathrm{ha}^{-1}$ promoveram a maturação precoce da cana-de-açúcar, incrementando o teor de sacarose no colmo antecipando em pelo menos 30 dias a colheita da cana.

A aplicação dos maturadores não proporcionou aumento significativo no teor de fibras em relação a testemunha a $0,21,30,45$ e 60 DAA para $E_{1}$ e $E_{2}$ (Tabela 3).

A aplicação dos maturadores não proporcionaram aumento significativo do brix com relação a testemunha na quase totalidade das épocas avaliadas, 
com exceção da realizada a 21 DAA (Tabela 4). Os maiores valores de Brix foram observados nos tratamentos aplicados em $\mathrm{E}_{2}$. Este comportamento demonstrou efeito aditivo do produto em apressar a maturação, e consequentemente a colheita da cana- de-açúcar, porém não foi significativo nas condições do experimento. Oliveira et al. (1993) revelaram que a aplicação de sulfometuron metil $\left(15 \mathrm{~g} \mathrm{ha}^{-1}\right) \mathrm{e}$ ethephon (480 $\left.\mathrm{g} \mathrm{ha}^{-1}\right)$ aumentaram o Brix em pelo menos $0,9 \%$.

Tabela 4. Dados de Brix (\%) da cana soca obtidos com a aplicação de dois maturadores em dois níveis de umidade do solo em seis épocas de avaliação. Jaguapitã, PR. 2001.

\begin{tabular}{|c|c|c|c|c|c|c|c|c|c|c|c|c|}
\hline \multirow[t]{2}{*}{ Tratamento } & \multicolumn{2}{|c|}{ O DAA } & \multicolumn{2}{|c|}{21 DAA } & \multicolumn{2}{|c|}{ 30 DAA } & \multicolumn{2}{|c|}{45 DAA } & \multicolumn{2}{|c|}{ 60 DAA } & \multicolumn{2}{|c|}{75 DAA } \\
\hline & Seco & Umido & Seco & Umido & Seco & Umido & Seco & Umido & Seco & Umido & Seco & Umido \\
\hline T1 & $13,4 \mathrm{a}$ & $14,0 \mathrm{a}$ & 14,2 & $15,0 \mathrm{a}$ & $15,5 \mathrm{a}$ & $15,2 \mathrm{a}$ & $14,9 \mathrm{a}$ & $15,4 \mathrm{a}$ & $15,2 \mathrm{a}$ & $15,6 \mathrm{a}$ & $15,4 \mathrm{a}$ & $15,5 \mathrm{a}$ \\
\hline & B & A & B & A & A & A & A & A & A & A & A & A \\
\hline $\mathrm{T} 3$ e $\mathrm{T} 4$ & $13,2 \mathrm{a}$ & $14,3 \mathrm{a}$ & $15,0 \mathrm{ab}$ & $15,3 \mathrm{a}$ & $16,2 \mathrm{a}$ & $16,1 \mathrm{a}$ & $15,7 \mathrm{a}$ & $15,8 \mathrm{a}$ & $15,9 \mathrm{a}$ & $15,9 \mathrm{a}$ & $15,4 \mathrm{a}$ & 16,2 \\
\hline & B & A & A & A & A & A & A & A & A & A & A & 4 \\
\hline T5 e T6 & $13,8 \mathrm{a}$ & $14,1 \mathrm{a}$ & $15,3 \mathrm{a}$ & $15,4 \mathrm{a}$ & $16,5 \mathrm{a}$ & $15,9 \mathrm{a}$ & $15,7 \mathrm{a}$ & $15,9 \mathrm{a}$ & $15,7 \mathrm{a}$ & $15,8 \mathrm{a}$ & $16,0 \mathrm{a}$ & $15,9 \mathrm{a}$ \\
\hline & A & 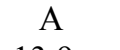 & & A & A & $\pi$ & A & A & A & 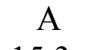 & A & 21 \\
\hline T7 e T8 & $\begin{array}{c}13,2 \mathrm{a} \\
\mathrm{B}\end{array}$ & $\begin{array}{c}13,9 \mathrm{a} \\
\mathrm{A}\end{array}$ & $\begin{array}{c}14,4 b c \\
B\end{array}$ & $\begin{array}{c}15,0 \mathrm{a} \\
\mathrm{A}\end{array}$ & $\begin{array}{c}15,4 \mathrm{a} \\
\mathrm{A}\end{array}$ & $\begin{array}{c}15,2 \mathrm{a} \\
\mathrm{A}\end{array}$ & $\begin{array}{c}15,0 \mathrm{a} \\
\mathrm{A}\end{array}$ & $\begin{array}{c}15,2 \mathrm{a} \\
\mathrm{A}\end{array}$ & $\begin{array}{c}15,9 \mathrm{a} \\
\mathrm{A}\end{array}$ & $\begin{array}{c}15,3 \mathrm{a} \\
\mathrm{A}\end{array}$ & $\begin{array}{c}15,0 \mathrm{a} \\
\mathrm{B}\end{array}$ & $\begin{array}{c}16,0 \mathrm{a} \\
\mathrm{A}\end{array}$ \\
\hline
\end{tabular}

Médias seguidas das mesmas letras minúsculas na coluna e maiúsculas na linha não diferem significativamente no nível de 5\% de probabilidade pelo Teste de Tukey.

Embora não registrados, foram observados, temporariamente, sinais leves de injúria às plantas de cana provocados pelos maturadores. Nos tratamentos com sulfometuron-methyl, os sintomas se caracterizavam por clorose das folhas centrais do cartucho e encurtamento de um a dois entrenós no ápice da planta, mas se recuperando e tendo seu desenvolvimento normal. Com ethephon, também ocorreu certo amarelecimento das folhas centrais do cartucho, encurtamento e expansão lateral de entrenós, porém os sintomas foram menos intensos.

\section{Conclusões}

Em função dos resultados obtidos e nas condições em que foi realizado o experimento, foi possível concluir que:

A ação dos maturadores aumentou significativamente com a umidade do solo a 0 e 21 dias após a aplicação;
Os aumentos de Pol\%, teor de fibra e Brix não foram significativos com a aplicação de sulfometuronmethyl e ethephon.

A aplicação de sulfometuron-methyl antecipou a colheita da cana soca em 15 dias.

\section{Referências}

CASAGRANDE, A. A. Tópicos de morfologia e fisiologia da cana-de-açúcar. Jaboticabal: FUNEP, 1991. 157p.

CASTRO, P. R. C. Ação comparada de maturadores em dois cultivares de cana-de-açúcar. Álcool \& Açúcar, São Paulo, n.73, p.36-39, 1994.

CASTRO, P. R. C.; MIYASAKI, J. M.; BEMARDI, M.; MARENGO, D.; NOGUEIRA, M. C. S. Efeito do ethephon na maturação e produtividade da cana-de-açúcar. Revista da Agricultura, Piracicaba, v.76, n.2, p.277-290, 2001.

CONSTANTIN, J.; MACIEL, C. D. G.; CONTIERO, R. L. Avaliação do uso de Sulfometuron-Methyl como maturador na cultura da cana-de-açúcar. In: CONGRESSO BRASILEIRO DA CIÊNCIA DAS PLANTAS DANINHAS, 22., 2000, Foz do Iguaçu. Resumos... Foz do Iguaçu: SBCPD, 2000.p.322. 
CORREAA, A. R.; GODOY, H.; BERNARDES, L. R. M. Características climáticas de Londrina. 2.ed. Londrina: IAPAR. 1982. 16p. (Circular IAPAR 5).

DEUBER, R. Maturação da cana-de-açúcar na região sudeste do Brasil. In: SEMINÁRIO DE TECNOLOGIA AGRONÔMICA, 4., 1988, Piracicaba. Resumo.... Piracicaba: Centro de Tecnologia COOPERSUCAR, 1988. p.33-40.

EMPRESA BRASILEIRA DE PESQUISA AGROPECUÁRIA (EMBRAPA). Sistema Brasileiro de Classificação de Solos. Rio de Janeiro: Centro Nacional de Pesquisa de Solos, 1999. 412p.

FERNANDES, J. Fatores de amadurecimento da cana-deaçúcar. Brasil Açucareiro, Rio de Janeiro, v.89, n.3, p.116122, 1977.

MARTINS, M. B. G.; CASTRO, P. R. C. Efeitos de giberilina e ethephon na anatomia de plantas de cana-de-açúcar. Pesquisa Agropecuária Brasileira, Brasília, v.34, n.10, p.1855-1863, 1999.

OLIVEIRA, D. A.; CASTRO, P. R. C. Efeito do Sulfometuron Methyl em cultura de cana-de-açúcar (Saccharum sp), cultivada em Latossolo vermelho-escuro, como maturador vegetal. In: CONGRESSO BRASILEIRO DE HERBICIDAS E PLANTAS DANINHAS, 19., 1993, Londrina. Resumos... Londrina: SBCPD, 1993.p.218.
OLIVEIRA, D. A.; CASTRO, P. R. C.; ANDRADE, T. L. C.; PONTIN, J. C.; PANINI, E. L.; DAMACENO, A.C.; SILVA, J. E.; MORAES JR., E. C.; VALÉRIO, W. G. Ação do maturador sulfemeturom metil na cana-de-açúcar cultivar SP70-1143. Revista Brasileira de Fisiologia Vegetal, Brasília, v.5, n.1,p.82, 1993.

ORGANIZAÇÃO DE PLANTADORES DE CANA DA REGIÃO SUL DO BRASIL (ORPLANA). Manual de Instruções da Consecana-SP-Anexo I. 6p. Disponível em: $<$ http: \|www.orplana.com.br/aruivos/anexoi.pdf $>$ Acesso em: 29/07/2005.

RODRIGUES, J. D. Fisiologia da cana-de-açúcar. Botucatu: UNESP:, 1995. 75p.

SALATA, J. C.; FERREIRA, L. J. Estudo da interferência do florescimento nas qualidades agroindustriais de algumas variedades de cana-de-açúcar. Brasil Açucareiro, Rio de Janeiro, v.88, n.6, p.19-24, 1977. 